\section{LA ÚLTIMA REFORMA DEL SISTEMA GENERAL DE SEGURIDAD SOCIAL EN SALUD EN COLOMBIA:

\author{
Lejano de la eficiencia y camino a la
} privatización total}

José O. López Oliva*

Fecha de Recepción: 22 de Abril de 2010

Fecha de Aceptación: 30 de Abril de 2010

Artículo de Reflexión

\section{Resumen}

La ley 1122 de 2007, mediante la cual se hacen algunas modificaciones al Sistema General de Seguridad Social en Salud en Colombia, refleja cambios encaminados a la privatización de los servicios de salud. Artículos como el No. 14 de la citada ley, tiene evidentes influencias del Código Comercial Colombiano en lo atinente a los actos y procedimientos médico-sanitarios; sumado a lo anterior, la Corte Constitucional señala que al delegarse la prestación del servicio

\footnotetext{
Abogado consultor, conferencista a nivel nacional e internacional, profesor Universitario de Pregrado y Postgrado, candidato a Magister en Derecho con enfoque en Derecho de la Responsabilidad y Seguros de la Universidad de los Andes, Magister de la Universidad Carlos III de Madrid (España), Especializado en Derecho Comercial de la Universidad Pontificia Bolivariana de Medellín; con estudios de postgrado en Derecho Penal y Probatorio de la Universidad del Rosario. Catedrático a nivel nacional dentro del programa de capacitación a verificadores de las condiciones de habilitación de prestadores de servicios de salud coordinado por el Ministerio de Protección Social. Docente certificado a nivel internacional por la Life Office Management Asociation, Inc - Loma con sede en Atlanta-Georgia (USA). Ha escrito y publicado textos de contenido jurídico. Profesor de la facultad de derecho de la Universidad Militar Nueva Granada. Correo electrónico: jose.lopez@unimilitar. edu.co ; joselopez@jloabogados.com
}

público de salud a una entidad particular, ésta ocupa el lugar del Estado legitimándose para obtener utilidades a costa de la salud de los usuarios de este servicio.

\section{Palabras Clave}

Salud, Privatización, Negocio, Ineficacia, Improvisación, SISBEN.

\section{THE LAST REFORM OF THE GENERAL SYSTEM OF SOCIAL SECURITY IN HEALTH IN COLOMBIA:}

Distant from the efficiency and near to the total privatization

\begin{abstract}
The 1122 law of 2007, that present some modifications to the general Colombian system of health, reflects changes aimed to the privatization of the health service. Some articles, such as the number 14 of this law, present evident influences from the Colombian commercial code concerning to the acts and procedures of the health care.
\end{abstract}

The constitutional court notes that the delegated provision of public health service to a particular institution takes the place of the State legitimizing to get profit at the expense of the health of service users

\section{Keywords}

Health, Privatization, Business, Inefficiency, Improvisation, SISBEN

\section{INTRODUCCIÓN}

El presente escrito, es resultado de un trabajo exploratorio relacionado con la última reforma expedida por el legislador, concretamente la Ley 1122 de 2007. En principio, el poder legislativo, en aras de establecer normas que regulen el Régimen de Seguridad Social en 
Colombia, expide la Ley 100 en el año de 1993; en su Artículo No. 157, esta determina dos vías para la afiliación al Sistema General de Seguridad Social en Salud ${ }^{1}$ (SGSSS): De un lado, el Régimen Contributivo, dirigido a las personas ${ }^{2}$ con contrato de trabajo, los servidores públicos, los pensionados y jubilados, y adicionalmente los trabajadores independientes que cuenten con una capacidad de pago. Por otro lado, el Régimen Subsidiado, adecuado para las personas que carecen de recursos económicos para asumir la totalidad del monto de cotización.

De la misma manera, la norma citada establece el acceso al SGSSS por medio de los denominados "participantes vinculados", quienes por motivos de incapacidad de pago (no por ello dejan de ser atendidos) y mientras logran ser beneficiarios del Régimen subsidiado, tienen derecho a los servicios de atención de salud que prestan las instituciones públicas y aquellas privadas contratadas por el Estado. ${ }^{3}$

Igualmente, se establecen los criterios de priorización ${ }^{4}$ de los beneficiarios de subsidios, a través de una lista que revela las prelaciones que deben imperar en los programas asistenciales hacia distintos grupos poblacionales, entre los

1 La Organización Mundial de la Salud, ha definido la salud como el: "estado en el que el ser orgánico ejerce normalmente todas sus funciones" o como simple "ausencia de enfermedad" y adicionalmente como: "el estado de completo bienestar físico, mental y social y no solo la ausencia de enfermedad y dolencias" véase: http://www.who.int/peh-emf/research/agenda/es/ index.html u.v. 20/05/09

2 Para efectos de este escrito se equiparan personas, individuos y población como lo mismo.

3 Ley 100 de 1993, citada y comentada en la Corte Constitucional, Sentencia T-441/06 de 02/06/06 expediente T- 1170394. Acción de tutela interpuesta por Ana Lucía Londoño Jaramillo y otras contra la Alcaldía Municipal de Manizales. M.P. Jaime Córdoba Triviño.

4 Consejo Nacional de Seguridad Social en Salud. Acuerdo 244/03 Artículo 7. que se cuentan los recién nacidos y las mujeres en estado de embarazo, entre otros. ${ }^{5}$

Según esto, la focalización del gasto público social depende del análisis de la información de los factores socioeconómicos y demográficos, tanto de los hogares como de las personas destinatarias de los beneficios. Para dicho efecto, el Estado $^{6}$ ha establecido un Sistema de Identificación y Clasificación de Potenciales Beneficiarios para Programas Sociales (SISBEN), mediante el cual se identifica y selecciona a los beneficiarios, con base en la información recogida de las personas entrevistadas ${ }^{7}$. Dichas entrevistas versan sobre temas relacionados con los servicios públicos, la educación de los miembros de la familia, la ocupación de los mismos y se verifica, entre otros datos, la calidad de las

$5 \quad$ Ibídem. La relación total es como sigue: 1 . Recién nacidos. 2. Menores desvinculados del conflicto armado, bajo la protección del Instituto Colombiano de Bienestar Familiar ICBF. 3. Población del área rural. 4. Población indígena. 5. Población del área urbana. Adicionalmente, para los grupos de la población descritos en los numerales 3, 4 y 5; al mismo tiempo, se fija en la norma, un nuevo criterio de prioridad para la afiliación a favor de los siguientes grupos: 1. Mujeres en estado de embarazo o período de lactancia que se inscriban en programas de control prenatal y posnatal. 2. Niños menores de cinco años. 3. Población con discapacidad identificada mediante la encuesta SISBEN. 4. Mujeres cabeza de familia. 5. Adultos mayores. 6. Población en condición de desplazamiento forzado. 7. Núcleos familiares de las madres comunitarias. 8 . Desmovilizados. Véase igualmente Corte Constitucional. Sentencia T-441/06 de 02/06/06. Ob. Cit.

6 Para efectos de este escrito tómese Estado, Gobierno y Nación como similares.

7 NÚÑEZ M, Jairo, et al. Evolución de las Politicas contra la Pobreza: De la previsión social a las transferencias condicionadas. Facultad Latinoamericana de Ciencias Sociales - FLACSO. Facultad de Economía. Universidad de los Andes. Bogotá. Documento CEDE 2006-31 issn 1657-7191 (edición electrónica) agosto de 2006. Este documento, según se indica en el mismo, "fue preparado para la Facultad Latinoamericana de Ciencias Sociales - FLACSO y hace parte de una publicación más extensa que recopila las experiencias de los países de América Latina que implementaron intervenciones de transferencias condicionadas basados en el programa Progresa- Oportunidades de México". 
viviendas donde habitan las personas encuestadas y si alguna de ellas está afiliada al SGSSS dentro del régimen contributivo. Con base en esta información, se establece desde el hogar más pobre hasta el menos pobre. ${ }^{8}$ Si bien es cierto, mediante el SISBEN se busca proteger a la población marginada (con los reparos que se presentan más adelante), y se expiden normas que tienden a afectar negativamente en algunos aspectos a la población en general.

En efecto, cada día la normatividad apunta a establecer una estructura privatista del SGSSS en Colombia. De hecho, existen normas que soportan la presente afirmación; en principio, a la luz de la Constitución Política, y posteriormente con la presentación de la Ley 1122 de $2007^{9}$, la cual modifica sustancialmente el régimen del SGSSS comentado ${ }^{10}$. Algunos ajustes, realizados a las instituciones del sistema, sin duda son relevantes para los afectados por los mismos ${ }^{11}$; ante todo, esta afectación se evidencia en la población pobre y vulnerable, la cual no cuenta con los recursos necesarios para suplir sus necesidades en salud. No obstante, disposiciones como el Artículo No. 14 de la Ley en mención, que habla "Del Aseguramiento", claramente dirigen el SGSSS hacia la prestación e intervención exclusiva por parte de entes

8 NÚÑEZ M, Jairo, et al. Evolución de las Políticas contra la Pobreza: De la previsión social a las transferencias condicionadas. Ob. Cit. pp. 7 y ss

9 Véase: Diario Oficial 46.506. Imprenta Nacional de Colombia. Ley 1122 del 9 de enero de 2007

10 RODRÍGUEZ SALAZAR, Oscar, Crecimiento, equidad y ciudadanía: Hacia un nuevo sistema de protección social. Universidad Nacional de Colombia, Facultad de Ciencias Económicas Centro de Investigaciones para del Desarrollo. Bogotá. 2006. p. 262. "La ley 100 de 1993 intenta plasmar el principio de que la República de Colombia está instituida como estado social de derecho $y$, por tanto, da vía a una nueva concepción de ciudadanía, articulada no sólo a los derechos políticos y civiles sino también al ejercicio de los derechos sociales".

11 El tema interesa a los usuarios del servicio de salud, a su recurso humano y a las autoridades públicas, entre otros. privados, que actúan como concesionarios del Estado en la prestación de este servicio.

Así las cosas, el siguiente escrito se aborda de la siguiente manera: en principio (i) se indica el concepto de Protección Social, para pasar a (ii) revelar algunas modificaciones realizadas a la Ley 100 de 1993 por la Ley 1122 de 2007; paralelamente, (iii) se trata el tema de los concesionarios de la salud y el sector privado, haciendo alusión al Artículo No. 14 de la citada ley. Posteriormente, (iv) se trata lo atinente al Régimen Subsidiado de Salud, donde se exteriorizan varios reparos a los procesos de encuesta y clasificación de las personas por medio del SISBEN. En igual sentido, (v) se enfatiza sobre la relevancia de la focalización del gasto público social de manera progresiva en el área de la salud, y para finalizar (vi) se critican los programas subsidiados por el Estado, se analiza (vii) una sentencia de la Corte Constitucional y se presentan (viii) unas breves conclusiones.

\section{CONCEPTO DE PROTECCIÓN SOCIAL}

El concepto de Protección Social que la Comisión Económica para América Latina y el Caribe (CEPAL) $)^{12}$ señala, al referirse a la necesidad imperiosa de establecer un pacto social para su observancia, se presenta en estos términos:

"La conformación de los estados de bienestar que surgieron en el mundo desarrollado entre los años treinta y sesenta del siglo $X X$, respondió a la búsqueda de sistemas de protección social ante los riesgos propios de la época, vinculados al papel

12 "La CEPAL es una de las cinco comisiones regionales de las Naciones Unidas y su sede está en Santiago de Chile. Se fundó para contribuir al desarrollo económico de América Latina, coordinar las acciones encaminadas a su promoción y reforzar las relaciones económicas de los países entre si y con las demás naciones del mundo. Posteriormente, su labor se amplió a los países del Caribe y se incorporó el objetivo de promover el desarrollo social". Disponible en:

http:/ /www.eclac.org/cgi-bin/getprod.asp? xml= / noticias/paginas/4/21324/P21324.xml\&xsl=/tp1/ p18f-st.xs1\&base=/tp1/top-bottom_acerca.xsl. 
central del trabajo en la definición y satisfacción de los derechos de las personas. Pero desde los años setenta del siglo XX en adelante, con los cambios que se fueron dando en la producción $y$ en el trabajo, en los perfiles epidemiológicos $y$ demográficos, en las familias y en los roles de género, quedaron cuestionados los Estados de bienestar, construidos en torno del trabajo (modelo bismarckiano). Esto llevó a plantear con fuerza la necesidad de reformas con vistas a proteger los derechos ciudadanos ante los nuevos riesgos sociales derivados de estos cambios, reformas que deben concebirse con gran cuidado para evitar la exclusión en la protección social (Titelman y Uthoff, 2005) ${ }^{13}$ (negrillas fuera del texto NFT) ${ }^{14}$.

Es importante reflexionar sobre las modificaciones que se realicen en torno al Sistema de Protección Social, concentrando dichos cambios en modelos que permitan la construcción de un nuevo concepto de ciudadanía basado en el ejercicio de los derechos sociales y que, además, proteja a la población colombiana de los riesgos inherentes a las sociedades de mercado. Es decir, buscar "un camino para que el orden económico satisfaga la necesidad del orden doméstico en el marco de un nuevo acuerdo social" 15 , y no para que el bienestar se presente en una sola vía, la de los operadores del sistema denominados Empresas Promotoras de Servicios de Salud (EPS), privadas, que como ya se dijo fungen como concesionarias de la salud.

13 Comisión Económica para América Latina y el Caribe CEPAL. "La protección social de cara al futuro". Trigésimo primer periodo de sesiones, Montevideo Uruguay, 2006, cap. I, pp 30 y ss. En CORTÉS GONZÁLEZ, Juan Carlos. Reforma al sistema de salud. Legis Editores S.A. Bogotá. 2007. p. xii

14 En el texto se utilizarán las siglas NFT que significan: negrillas fuera del texto.

15 RODRIGUEZ SALAZAR, Oscar, Crecimiento, equidad y ciudadanía: Hacia un nuevo sistema de protección social. Ob. Cit. p. 189.

\section{LA LEY 100 DE 1993 Y ALGUNAS MODIFICACIONES REALIZADAS POR LA LEY 1122 DE 2007}

Con la expedición de la Ley 100 de 1993, es claro que se cambian, entre otros, la relación entre los profesionales de la salud con los denominados "pacientes" o usuarios de este servicio. Esta norma fue modificada por la Ley 1122 de 2007, la cual tiene como objeto:

"realizar ajustes al Sistema General de Seguridad Social en Salud, teniendo como prioridad el mejoramiento en la prestación de los servicios a los usuarios. Con este fin se hacen reformas en los aspectos de dirección, universalización, financiación, equilibrio entre los actores del sistema, racionalización, y mejoramiento en la prestación de servicios de salud, fortalecimiento en los programas de salud pública y de las funciones de inspección, vigilancia y control y la organización y funcionamiento de redes para la prestación de servicios de salud"16 (NFT).

Con esta ley se busca, en principio, minimizar el riesgo en salud así como el examen de la calidad en la prestación del servicio; finalmente, el reto se enfoca en el aseguramiento de toda la población colombiana, en lo que se ha denominado la "Universalidad" 17 .

Se presentan a continuación algunos cambios significativos con que cuenta la Ley 1122, se comentan dichas modificaciones y se hacen los reparos pertinentes a esta.

\subsection{Comisión de regulación en salud}

En la Ley 1122 se instituye la denominada Comisión de Regulación en Salud (CRES),

16 Ley 1122 de 2007. Artículo 1.

17 La Universalidad es un principio de la Ley 100 del 1993 que rige el Sistema de Seguridad Social en Salud, junto con la Solidaridad, Unidad e Integralidad. El Estado propende por la salud de todas las personas mediante el principio de Universalidad que, igualmente, se encuentra consagrado en la C.P. con la consecuente NO limitación a los ciudadanos de los programas asistenciales en salud. 
pero se aclara que el Consejo Nacional de Seguridad Social (CNSSS) no desaparece y, por el momento, se le otorgan labores de asesoramiento y consultoría; entre las más relevantes funciones de la CRES está la del establecimiento del Plan Obligatorio de Salud (POS). Una de las críticas que se presentan en relación con este punto, es que dichos comisionados son escogidos y nombrados por el Presidente de la República, con la consecuente desaparición de la participación de la "ciudadanía, (quien debería tener) la posibilidad de ejercer los derechos politicos" 18, y así garantizar los derechos civiles y sociales de la comunidad ${ }^{19}$.

\subsection{El defensor del usuario en salud}

Uno de los aspectos positivos de la Ley, es la instauración del defensor del paciente (usuario del servicio en salud) que está bajo la coordinación de la Superintendencia Nacional de Salud, aunque sería primordial que este estuviera bajo la dirección de la Defensoría del Pueblo, porque esto generaría mayor credibilidad e independencia.

\subsection{Disminución del tiempo de cotización y periodo de carencia}

Ciertamente, el hecho de que se haya disminuido a un año el tiempo de cotización a una EPS, para contar con la posibilidad del traslado a otra Entidad Prestadora del Servicio, puede ser benéfico; también, la respectiva rebaja en el tiempo del período de carencia a 26 meses para las enfermedades de tratamiento quirúrgico y las de alto costo $^{20}$, como por ejemplo el SIDA o los procedimientos de diálisis.

18 RODRÍGUEZ SALAZAR, Oscar, Crecimiento, equidad y ciudadanía: Hacia un nuevo sistema de protección social. Ob. Cit. p. 202.

19 Ibídem.

20 El Congreso de la República, hay que decirlo, no ha estado ajeno a esta realidad que afecta, ante todo, a la población más pobre. "Las Comisiones Séptimas de

\subsection{Medidas que ayudan a la capitalización del SGSS}

La Ley amplía la cotización al régimen contributivo en $0.5 \%$ y el porcentaje del Sistema General de Participaciones que los entes territoriales deben destinar a la afiliación en el Régimen Subsidiado, disminuyendo proporcionalmente el aporte del gobierno nacional. "Se afirma que no se alcanzará la cobertura universal al no estar en ninguno de los regímenes la población de 19 a 24 años desempleados, no estudiantes y solteros, que se calcula en 2'000,000 de personas y la población no afiliada al régimen contributivo del nivel III del Sisben" 21.

No obstante, las modificaciones realizadas al SGSSS en Colombia no muestran una estructura jurídica y económica sólida y coherente, porque si bien es cierto que se indica, por ejemplo, que la salud de las personas debe prevalecer $^{22}$, muchos de los recursos destinados

Senado y Cámara aprobaron que las EPS puedan atender usuarios que padezcan enfermedades de alto costo, a través de la contratación de un seguro. Igualmente, le dieron el visto bueno a 150 mil millones de pesos para la prestación de servicios a la población pobre, los cuales se utilizarán por una sola vez de los excedentes acumulados a diciembre del 2005, de la subcuenta de Eventos Catastróficos y Accidentes de Tránsito, ECAT, del Fondo de Solidaridad de Garantías. De acuerdo con el artículo aprobado, los recursos se distribuirán entre las entidades territoriales y las EPS, conforme a los criterios que para tal efecto defina el Ministerio de la Protección Social y se ejecutarán a través de una cuenta especial en el respectivo fondo de salud a nombre del proyecto de atención prioritaria en salud nacional". Véase: Senado de la República de Colombia, Oficina de Información y Prensa, 14 de noviembre de 2006. Disponible en: http://servoaspr.imprenta. gov.co:7778/gacetap/gaceta.mostrar_documento?p_ tipo $=01 \&$ p_numero $=06 \&$ p_consec $=16949$

21 TAFUR, Luis Alberto. Controversia a la reforma de la Ley 100 de 1993, Ley 1122 de enero de 200. Corporación Editora Médica del Valle. Universidad del Valle. Cali. 2007.

22 Constitución Política de Colombia. Artículo 336 ordena: "(...)...Las rentas obtenidas en el ejercicio de los monopolios de suerte y azar estarán destinadas exclusivamente a los servicios de salud." De la misma manera: "Los recursos del Sistema General de Participaciones de los departamentos, distritos y municipios se destinarán a la financiación de los servicios a su cargo, dándole prioridad 
a este sector se desvían para la ejecución de otras actividades. El deber de todos los Estados al establecer y estructurar los SGSSS $^{23}$ es el de prevención, atenuación y corrección de las fallas de dichos sistemas, en el marco de los principios de igualdad y equidad ${ }^{24}$, destinados a los asociados que claman por su derecho fundamental a la salud.

\section{CONCESIONARIOS DE LA SALUD: EL SECTOR PRIVADO}

La presente exposición, como lo anotamos con anterioridad, se enfoca en la ineficiencia del SGSSS, el cual se encamina a su privatización total. Para este efecto, en principio se hace referencia a las normas constitucionales pertinentes, para pasar en seguida a analizar la Ley 1122 referida, concretamente el Artículo No. 14.

La Constitución Política de Colombia, en su Artículo No. 48, indica:

"La Seguridad Social es un servicio público de carácter obligatorio que se prestará bajo la dirección, coordinación y control del Estado, en sujeción a los principios de eficiencia, universalidad y solidaridad, en los términos que establezca la Ley. Se garantiza a todos los habitantes el derecho irrenunciable a la Seguridad Social. El Estado, con la participación de los particulares, ampliará progresivamente la cobertura de la

al servicio de salud y los servicios de educación preescolar, primaria, secundaria y media, garantizando la prestación de los servicios y la ampliación de cobertura".

23 Constitución Política de Colombia. Artículo 2: "Son fines esenciales del Estado: servir a la comunidad, promover la prosperidad general y garantizar la efectividad de los principios, derechos y deberes consagrados en la Constitución... Las autoridades de la República están instituidas para proteger a todas las personas residentes en Colombia, en su vida, honra, bienes, creencias, y demás derechos y libertades, y para asegurar el cumplimiento de los deberes sociales del Estado y de los particulares" (NFT).

24 La Equidad es la "realización suprema de la justicia, que a veces va más allá de lo que prescribe la ley". Véase: GUILLEN, Raimon, et al. Diccionario Jurídico. Temis. Bogotá. 1990. p. 168.
Seguridad Social que comprenderá la prestación de los servicios en la forma que determine la Ley"25.

Y continúa la mencionada norma: "La Seguridad Social podrá ser prestada por entidades públicas o privadas, de conformidad con la ley. No se podrán destinar ni utilizar los recursos de las instituciones de la Seguridad Social para fines diferentes a ella. La ley definirá los medios para que los recursos destinados a pensiones mantengan su poder adquisitivo

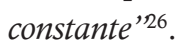

De igual manera, el Artículo 49 de la Constitución Nacional señala: “(...) El Estado, con la participación de los particulares, ampliará progresivamente la cobertura de la Seguridad Social que comprenderá la prestación de los servicios en la forma que determine la Ley. La Seguridad Social podrá ser prestada por entidades públicas o privadas, de conformidad con la ley. (...)”27 (NFT).

Es evidente en los anteriores artículos que la misma Constitución Política delega en particulares su deber de asistencia en salud. Por ello, el sector privado ha efectuado grandes inversiones con el fin de desarrollar sus objetos sociales, destinados a la prestación de servicios de salud. Ante esto, el Estado por medio de sus instituciones $^{28}$, debe estar vigilante y presto a tomar los correctivos necesarios, para que la asistencia en salud se preste de una manera eficiente y con calidad.

Una vez indicadas y brevemente analizadas las normas de la carta constitucional, se analiza

25 Disponible en: http://www.secretariasenado.gov.co/ senado/basedoc/cp/constitucion_politica_1991_ pr001.html

26 Constitución Política. Artículo 48.

27 Disponible en: http://www.secretariasenado.gov.co/ senado/basedoc/cp/constitucion_politica_1991_ pr001.html

28 Ministerio de Protección Social, Secretarías Departamentales y Municipales de Salud, Superintendencias, en especial la Nacional de Salud, entre otras. 
Tabla 1.

\begin{tabular}{cccccc}
\hline$\underline{\text { Subsidio Pleno }}$ & $\underline{\text { Subsidio Parcial }}$ & Contributivo & $\underline{\text { Especiales }}$ & Población Nocubierta & Población Total \\
\hline 18.381 .731 & 279.679 & 15.533 .582 & 2.015 .186 & 5.032 .770 & 41.242 .948 \\
\hline
\end{tabular}

concretamente el Artículo 14 de la Ley 1122 que señala:

"Para efectos de esta ley entiéndase por aseguramiento en salud, la administración del riesgo financiero, la gestión del riesgo en salud, la articulación de los servicios que garantice el acceso efectivo, la garantía de la calidad en la prestación de los servicios de salud y la representación del afiliado ante el prestador y los demás actores sin perjuicio de la autonomía del usuario. Lo anterior exige que el asegurador asuma el riesgo transferido por el usuario $y$ cumpla con las obligaciones establecidas en los Planes Obligatorios de Salud. Las Entidades Promotoras de Salud en cada régimen son las responsables de cumplir con las funciones indelegables del aseguramiento. Las entidades que a la vigencia de la presente ley administran el régimen subsidiado se denominarán en adelante Entidades Promotoras de Salud del Régimen Subsidiado (EPS). Cumplirán con los requisitos de habilitación y demás que señala el reglamento" ${ }^{29}$ (NFT).

Se demuestra palmariamente en el mencionado Artículo, que el denominado "Aseguramiento" es una noción que trae consigo toda una estructura de intermediación privada de los recursos en salud que, sin duda, encarecen la prestación de este servicio, ahondando las crisis del aseguramiento social -que se expresan en bajas coberturas, inequidad, problemas financieros y fiscales-, y con mayor presencia de los grupos económicos en el SGSSS. ${ }^{30}$

29 Disponible en: http://www.secretariasenado.gov.co/ senado/basedoc/ley/2007/ley_1122_2007.html

30 RODRÍGUEZ SALAZAR, Oscar, Crecimiento, equidad y ciudadanía: Hacia un nuevo sistema de protección social. Ob. Cit. p.198.
Al observar algunos estudios realizados por el Ministerio de Protección Social, se advierte que la "equidad" está ausente. Estas cifras son garantes de lo afirmado:

Análisis de cobertura 2005 - $2006^{31}$ (Tabla 1.)

"El cuadro anterior, denota la ausencia de equidad en el sistema, ya que 5.032.770 Colombianos no tienen garantizado el acceso a los servicios de salud y que realmente solo el $43 \%$ de los colombianos, los afiliados a los Regimenes Contributivo y Especiales, tienen acceso a todos los niveles de atención contemplados en el Sistema, ya que un plan Subsidiado, corresponde al 56\% de un POS Contributivo y un Subsidio Parcial a tan solo el $23 \% " 32$.

Con el mismo propósito, la Gaceta del Congreso Número 596 con fecha 30 de noviembre de 2006 indica:

"Las anteriores cifras resultan más preocupantes si se tiene en cuenta que los recursos públicos destinados al sector salud, se han venido incrementando de forma acentuada a partir del año 1993, pasando de un $2 \%$ a un $4 \%$ del PIB, es decir, un incremento de $100 \%$ en 9 años" 33 (NFT).

Y continúa: "En materia de recursos, los mismos son administrados por el Fosyga, sobre los cuales la Contraloría General de la República,

31 Presentado por el Ministerio de Protección Social. Fuente: MPS, Informe de Actividades 2005-2006. Disponible en: www.minproteccionsocial.gov.co uv. 20/05/09.

32 Gaceta del Congreso No. 596. 30 de noviembre de 2006.

33 Disponible en: http://servoaspr.imprenta.gov.co:7778/ gacetap/gaceta.mostrar_documento?p_tipo $=11 \&$ p_ numero $=040 \&$ p_consec $=14498$ 
en auditoría integral gubernamental de 2004 concluye que: $\mathrm{El}$ resultado obtenido revela que el Fondo de Solidaridad y Garantía, Fosyga, como Cuenta adscrita al Ministerio de la Protección Social, presenta debilidades de control en los sistemas contables, financieros, de planeación, de información y operacionales, que influyen en la ejecución eficiente y oportuna de los recursos que administra, lo que ha permitido, según el informe de auditoría, que recursos cuantificados en \$1,4 billones, no se hayan aplicado adecuadamente dentro del Sistema General de Seguridad Social en Salud" ${ }^{\prime 34}$ (NFT).

De otro lado, la noción de "Aseguramiento", en concreto de la Protección Social en Salud, está evidentemente influenciada por el Derecho Comercial ${ }^{35}$ porque, entre otras, se descubren y aplican conceptos como los de Riesgo (contingencia en materia de seguridad social) definido por la legislación mercantil, como el suceso incierto, cuya realización da origen a la obligación del Asegurador ${ }^{36}$ (en salud en este caso). El citado Asegurador es quien asume el Riesgo a cambio del pago de una Prima, definida como el importe a cargo del asegurado, por el hecho de trasladar los riesgos, como la enfermedad, por ejemplo; en el campo de la salud dicha Prima, se coteja con la Unidad de Pago por Capitación. Al mismo tiempo, se muestra un tomador, quien es parte dentro del Contrato de Seguro y traslada los riesgos a una Compañía Aseguradora.

Es de anotar, que dentro del Régimen Contributivo el Tomador es el empleador, y si el trabajador es independiente, este hace sus veces; contrario sensu en el Régimen Subsidiado, en el que el

34 http://www.alcaldiabogota.gov.co/sisjur/normas/ Norma1.jsp?i=21344

35 El Código de Comercio Colombiano, establece todo lo atinente al contrato de seguros en las normas contenidas desde los Artículos 1036 al 1162.

36 Código de Comercio. Artículo 1054 tomador es el Estado ${ }^{37}$. Se refleja, por último, un Asegurado quien cuenta con una cobertura definida como el conjunto de prestaciones a cargo del Asegurador, que deben ser cubiertas en el evento de la realización del riesgo, y que en salud se equiparan a los Planes de Beneficios o Plan Obligatorio de Salud (POS). No solo es que se traten, se ratifica, de simples conceptos importados del Código de Comercio a las regulaciones en salud; el hecho es, que hay visos de una relación contractual donde el Estado "no interviene" y puede ser plausible, como ya está pasando, que dicha relación contractual se maneje bajo las nociones de "parte dominante" (Aseguradora) y "parte débil" (Usuario); en esta relación a este último le correspondería "adherirse" a los condicionamientos impuestos por la Aseguradora. De esta forma se evidencia uno de los grandes problemas del sistema, para pasar ahora a analizar los inconvenientes que se presentan en el SISBEN.

\section{EL SISBEN Y LA IDENTIFICACIÓN DE LA POBLACIÓN POBRE Y VULNERABLE}

E1 SISBEN es una herramienta propia del Estado, con la que se prevé administrativamente la identificación de la población pobre y vulnerable, la cual recibirá los subsidios a cargo de aquel. En síntesis, con base en este proceso de medición a cargo de los entes territoriales ${ }^{38}$, se determinan los variados programas de promoción a cargo del Estado; por ejemplo, en el área de la salud, desde ya se indica que debe tener preponderancia sobre los demás programas

37 CORTÉS GONZÁLEZ, Juan Carlos. Reforma al sistema de salud. pp. 140 y ss

38 Como son los Departamentos y Municipios. Igualmente, véase: Ley 715 de 2001. Artículo 94 que indica lo atinente a la disposición de los recursos destinados al gasto público, los cuales se deben ejecutar por medio de instrumentos que permitan la identificación de la población pobre y vulnerable. Dichos criterios de identificación los determina de forma regular el Consejo Nacional de Política Económica y Social Conpes. 
asistenciales. Ahora bien, son evidentes los numerosos inconvenientes que presentan estos procesos de identificación.

¿Cómo se identifica entonces a la población pobre y vulnerable? Son dos los instrumentos establecidos por el Consejo Nacional de Política Económica y Social (CONPES) a saber: de un lado, los instrumentos de focalización geográfica, y del otro, los instrumentos de focalización individual.

\subsection{Instrumentos de focalización geográfica}

La focalización geográfica se concentra en la identificación de las áreas donde se agrupa la población pobre, con base en las características tanto de la vivienda como de su entorno, con el fin de aplicar a todos sus habitantes programas sociales de índole colectivo, entre los que se cuenta infraestructura, dotación y saneamiento ambiental, al igual que la implementación de políticas de diferenciación en las tarifas para el pago de servicios públicos domiciliarios ${ }^{39}$. De esta forma, la focalización geográfica permite la ejecución de programas de beneficio general, esto es, "que todos los habitantes de un estrato se beneficien por igual del desarrollo y ejecución de programas generales de infraestructura y de menores tarifas para el pago de servicios públicos (subsidiados: estratos 1 a 3$)^{\prime \prime 40}$.

E1 reparo que se presenta en este instrumento de focalización, se resume en que únicamente se tienen en cuenta las características exteriores de las viviendas y el desarrollo del sector objeto de la identificación. Aquí nacen, por ello, una serie de interrogantes: ¿habitar en una zona determinada refleja necesariamente las condiciones económicas de la persona? ¿El estilo de vida de un individuo indica su status

\footnotetext{
39 Véase: http://sisconpes.dnp.gov.co/AYUDA/CON SULTADEINFORMACI\%c3\%93N/DocumentosConpesAprobados/tabid/131/Default.aspx

$40 \quad$ Ibídem
}

económico? En síntesis, esta forma de identificación, al centrarse en características físicas exteriores de las viviendas del sector y del nivel de desarrollo de su entorno, hace que los recursos escasos del ente Estatal no se dosifiquen de manera adecuada.

Inquieta, en efecto, este instrumento de focalización geográfica por cuanto refleja un alto nivel de generalidad, dado que si este se aplica en una zona rural donde sus habitantes son campesinos de escasos recursos, y adicionalmente existen predios en esa zona dedicados a la explotación agropecuaria, esto no significa que "todos" los que estén asentados en dicho territorio deban ser beneficiados por los programas del gobierno. Habrá individuos que son propietarios de extensos predios y que con seguridad no requieren de la asistencia del Estado; esos recursos podrían ser destinados, en definitiva, a la población verdaderamente pobre y vulnerable, primordialmente para los servicios de salud ${ }^{41}$.

\subsection{Instrumentos de focalización individual}

La focalización individual tiene, igualmente, por instrumento de implementación al SISBEN con el fin de identificar y clasificar en seis niveles a la población más pobre y vulnerable, "con base en la información relacionada con educación, hacinamiento, calidad de la vivienda, su equipamiento $y$ servicios, aspectos demográficos, el ingreso, la ocupación y la seguridad social'"42. La intención de este instrumento es, ante todo, identificar los hogares e individuos pobres que, por sus especiales características socioeconómicas y de vulnerabilidad, merecen ser incluidos en los programas

${ }_{41}$ CURREA, Lugo. La Salud como Derecho Humano. España. 2005. p.23. ROMERO, Coloma. La Medicina. España. 2002. p.117. Estos dos autores tratan sobre la preeminencia con que debe contar el servicio de salud $y$ en el que se deben enfocar los Estados.

42 Véase: http://sisconpes.dnp.gov.co/AYUDA/CON SULTADEINFORMACI\%c3\%93N/DocumentosConpesAprobados/tabid/131/Default.aspx 
sociales a cargo del Estado. Por tanto, lo que pretende el SISBEN con este instrumento es (i) identificar a las familias e individuos que posean las condiciones expuestas, así como (ii) otorgar, en un marco de crecientes restricciones presupuestales, los subsidios del Estado en condiciones de equidad, bajo la premisa de la atención prioritaria a los más pobres. Asimismo, se busca (iii) conocer los niveles de pobreza de las poblaciones más deprimidas, como herramienta valiosa para la planeación local y (iv) fomentar la inclusión social de los diferentes grupos de la población ${ }^{43}$. Se evidencia que existen variadas falencias en la aplicación de este instrumento, tal como se indicará más adelante, con el análisis de la sentencia de la Corte Constitucional.

\subsection{La igualdad y el igualitarismo}

En principio, resulta pertinente indicar que el SISBEN como instrumento de medición debe ser utilizado para la búsqueda de la igualdad, mas no del igualitarismo. Este último se expresa, de hecho, cuando el Estado no tiene en cuenta las circunstancias especiales y particulares de las personas, sino que estas cuentan con un idéntico tratamiento por parte de aquél ${ }^{44}$. En tal sentido, Colombia ha acogido protocolos como el de San Salvador, que se apega a la protección de los Derechos Humanos y concretamente a los Económicos, Sociales y Culturales; así, el Artículo No. 03 de dicho acuerdo enseña lo siguiente: "Los Estados partes en el presente protocolo se comprometen a garantizar el ejercicio de los derechos que en él se enuncian, sin discriminación alguna por motivos de raza, color, sexo, idioma, religión, opiniones politicas o de cualquier otra indole, origen nacional o social, posición económica, nacimiento o cualquier

\footnotetext{
$43 \quad$ Ibídem.

44 OLANO GARCÍA, Hernán Alejandro. Constitución Politica de Colombia. Ediciones Doctrina y Ley Ltda. Bogotá. p. 68
}

otra condición social" " ${ }^{45}$. Con lo anterior, sale a la luz el igualitarismo, más no la igualdad.

Contrario sensu se revela el principio cardinal de la igualdad, evidenciándose que "es a partir del tratamiento desigual que se contiene la igualdad" 46. Este derecho por consiguiente, "se traduce en la necesidad de tratar a todos de manera diferente a partir de sus especiales condiciones" 47 . Con todo, se deduce que una "diferenciación es discriminatoria si carece de justificación objetiva y razonable, es decir, si no persigue un fin legítimo o si carece de una relación razonable de proporcionalidad entre los medios empleados y el fin perseguido" 48. En consecuencia la inspiración aristotélica de que hay que tratar igual a lo igual, y desigual a lo desigual carece de utilidad ${ }^{49}$, por ejemplo, en lo atinente a los servicios prestacionales en favor de los pobres.

\section{LA PREEMINENCIA DEL SISTEMA DE SEGURIDAD SOCIAL EN SALUD}

Algunos programas asistenciales del Estado han resultado fallidos, como es el caso del Instituto Colombiano de Reforma Agraria (INCORA) ${ }^{50}$, donde se perdieron una infinidad de recursos que pudieron haber sido aprovechados para la atención sanitaria a la población pobre y vulnerable, que según la tesis que se presenta en este trabajo, debe preponderar. Es claro que otras

45 Véase: Ley 319 de 1996, mediante la cual Colombia se acoge al Protocolo de San Salvador.

46 OLANO GARCIA, Hernán Alejandro. Constitución Politica de Colombia. Ob. Cit. p. 68.

47 Ibídem.

48 Corte Europea de Derechos Humanos en: http:// www.echr.coe.int/echr/Homepage u.v. 19/05/09

49 OLANO GARCÍA, Hernán Alejandro. Constitución Politica de Colombia. Ob. Cit. p. 32.

50 Periódico El Tiempo, artículo denominado: “Tierra Movediza". Fecha de publicación 11/12/1997. Autor Unidad Investigativa. Este artículo refleja claramente la aparente corrupción a que han sido sometidos entes estatales como el Instituto Colombiano para la Reforma Agraria (INCORA) lo que ha llevado a su liquidación. 
entidades han corrido con la misma suerte, y en el área específica de la salud, la liquidación del Instituto Colombiano de Seguros Sociales (ISS) es una muestra palpable de la ineficiencia del Estado, en el manejo de este servicio prestacional especialmente relevante en las comunidades marginadas $^{51}$.

Resulta imprescindible anotar que, si bien es cierto, existen beneficios sociales que son importantes y el escenario primordial para el otorgamiento de dichas ayudas debe ser, sin vacilación, la afiliación al Régimen Subsidiado del SGSSS y el suministro de verdaderas prestaciones médico asistenciales a la población vinculada a este sistema. Además, se debe dar primacía al régimen asistencial en salud y concretarse en los sujetos de especial protección constitucional, como son los niños, discapacitados, adultos mayores, madres cabeza de familia y mujeres en estado de embarazo, entre otros. En conclusión, si no hay salud, no hay ni vivienda, ni educación, ni recreación, ni trabajo, razón por la cual es inminente que los recursos escasos estatales se dirijan a este sector.

\section{LOS PROGRAMAS ASISTENCIALES PARA LA POBLACIÓN POBRE Y VULNERABLE COMO INCENTIVO PARA SEGUIR EN ESTA SITUACIÓN}

Con los programas asistenciales subsidiados por el Estado en todas sus modalidades, se generan incentivos en la población identificada como pobre y vulnerable; lo anterior, genera la concepción de que "ser pobre es conveniente", por cuanto si se deja de serlo se suspenden los beneficios. Es importante que el ente estatal, según esto, genere otro tipo de incentivos con los cuales las personas marginadas traten de salir de esa cultura perversa de la consecución

$51 \quad$ En la actualidad la prestación de servicios en salud se presta por parte de la Nueva EPS. de auxilios; por ejemplo, monetarios que fundan "un país de mendigos y de limosneros" 52 .

En este orden de ideas, el Estado debe establecer incentivos para que los individuos salgan de la pobreza, y no para que se mantengan en ella con el objetivo de continuar ininterrumpidamente recibiendo el apoyo de aquél. Autores como Amartya $\mathrm{Sen}^{53}$, economista Indio ganador del

52 FRESNEDA, Oscar. Entrevista realizada al experto en Politica Social en la revista Salud Colombia. Public Health Journal on the net. Edición 55, Febrero 2001. Disponible en: http://www.saludcolombia.com/actual/ salud55/portad55.htm última visita: 26/04/09

53 "Amartya Sen [1999, 74-76] en su discusión sobre el desarrollo y la libertad ha distinguido los conceptos de capacidades $y$ de logros realizables. Logros realizables se refieren a las diversas condiciones de vida (las diversas dimensiones del ser y el hacer) que pueden o no ser alcanzadas, mientras que capacidades se refiere a nuestra habilidad para alcanzar dichas condiciones de vida. Una realización es un logro, mientras que una capacidad es la habilidad para lograr. Los logros realizables están, en cierto sentido, más ligados con las condiciones de vida, las capacidades en contraste, son nociones de libertad en el sentido positivo del término: las oportunidades reales que se tienen respecto a la vida que se puede llevar. La libertad para alcanzar diferentes tipos de vida se refleja en un conjunto de capacidades de la persona $y$ estas dependen de una variedad de factores, incluidas las caracteristicas personales y factores sociales. Una medición completa de la libertad debe por supuesto, estar basada en las capacidades de la vida personal y prestar atención a otros objetivos de la persona (por ejemplo, metas sociales que no estén directamente relacionadas con su propia vida). Las capacidades humanas para Sen constituyen una parte importante de la libertad individual. El concepto de libertad no está exento de problemas. Por ejemplo, dice Sen [1999, 74-76], si no tenemos el valor para escoger una forma de vida particular, aunque pudiéramos vivir de esa forma si la escogiéramos, es como si no tuviéramos la libertad de vivir de esta forma con la correspondiente capacidad. La expresión libertad, en ocasiones debe ser evaluada independientemente de los valores y preferencias de la persona cuya libertad se está determinando. Entonces la preocupación debe ser el rango de escogencia que una persona tiene, y no cómo ella evalúa los elementos en este rango, o qué escoge ella dentro del rango...". Disponible en: HERNÁNDEZ, Diego. Ph.D. Profesor Asociado, y Director del Departamento de Ingeniería de Sistemas e Industrial, Universidad Nacional de Colombia. Trabajo de investigación realizado con la colaboración de los profesores Jorge I. Bula, Jhon Betancur del College of Urban Planning and Public Affaires at University of Illinois in Chicago, COLCIENCIAS, la Universidad Nacional de 
Premio Nóbel de Economía en 1988, así como Philippe Van Parijs, "enfatizan en la importancia de la <libertad real> para que los individuos puedan ser capaces de agenciar su propio plan de vida, y optar en sus diferentes etapas...por la mejor opción dentro de una canasta de oportunidades de vida en vez de canasta de bienes, logrando con ello mejorar la calidad de vida de las personas, y el de la sociedad en su conjunto" ${ }^{24}$. No se trata, por ende, de suspender los incentivos del gobierno nacional otorgados a la población pobre y vulnerable; no obstante, se insiste en la generación de auxilios para las personas que traten de salir de ese estado de carencia continua, mediante acciones positivas centradas en un proyecto de vida, todo esto obviamente con la ayuda del Estado.

\section{EL SISBEN Y LA CORTE CONSTITUCIONAL}

En un fallo de tutela proferido por la Corte Constituciona ${ }^{55}$, diez y siete religiosas de una

Colombia y el College of Urban Planning and Public Affaire de la University of Illinois. Disponible en: www.docentes.unal.edu.co/dfhernandezl/docs/ Universalism_SocialPolicy_DFHL.pdf - uv.15/05/07

54 Ibídem.

55 Corte Constitucional. Sentencia SU 480 del 25/11/97. M.P. Alejandro Martínez Caballero. Se presenta a continuación, un breve recuento tanto de los hechos base de la tutela en comento, como de la decisión respectiva: "Ana Lucía Londoño Jaramillo y otras diez y seis Hermanas pertenecientes al Monasterio de la Visitación Santa Maria de Manizales, fueron beneficiarias durante varios años de la atención en salud ofrecida por la red pública de salud del municipio de Manizales, debido a su pertenencia al registro del sistema de selección de beneficiarios de programas sociales. Sin embargo, de forma intempestiva les fueron suspendidos los servicios en razón de lo que las actoras calificaron como "anulación" de los carnés del sistema de salud y la consecuente negativa a la prestación del servicio médico. Ante tal circunstancia, se dirigieron al Alcalde de la ciudad manifestándole la precaria situación en que se encontraban y la necesidad que fueran reincorporadas en el registro del sistema de selección de beneficiarios los de programas sociales - Sisben. Resaltaron que algunas de las Hermanas estaban enfermas y que, por ello, la suspensión de los servicios de salud podría tener graves consecuencias en su integridad fisica, amén de la falta de recursos económicos comunidad cristiana y beneficiarias de los programas asistenciales en salud resultaron marginadas, justificadamente según el fallo, de los servicios médico asistenciales bajo las disposiciones del Régimen Subsidiado de Salud; esto por el hecho de que dicha comunidad NO hacía parte de la población pobre y vulnerable. Es así como se refleja claramente que las prioridades del gasto público y el adecuado manejo de los recursos escasos con que cuenta el Estado, deben ser orientadas a los beneficiarios cuyo estrato socioeconómico requiera necesariamente de dichos servicios asistenciales; con preeminencia, se insiste, en la atención de los servicios de salud.

Al mismo tiempo, se demostró en el mencionado proceso, mediante un cuestionario

de la comunidad para asumir directamente los tratamientos médicos correspondientes. Luego de la remisión hecha por el Alcalde municipal a la Coordinación del Sisben, la Secretaría de Planeación de Manizales les informó a las demandantes que según los lineamientos metodológicos del Departamento Nacional de Planeación, no es posible realizar la aplicación de encuestas SISBEN a ninguna Institución, para el caso que nos ocupa el Monasterio de la Visitación de Santa María; esto en consideración que la unidad de medida para la aplicación de la encuesta era la vivienda, por lo que no aplicaba para hogares colectivos. Agregó que según lo previsto en el artículo $7^{\circ}$ del Decreto Municipal 0135 de 2004, la encuesta Sisben administrada desde 1994 habia perdido efecto. Con base en lo anterior, el 18 de mayo de 2005 las Hermanas interpusieron acción de tutela, pues consideraron que se vulneraban sus derechos constitucionales a la salud y a la seguridad social debido a la interrupción del servicio de salud subsidiada... En consecuencia, pretenden que le sea ordenado al ente municipal que las incorpore en el Régimen Subsidiado de Seguridad Social en Salud... " La Corte indica, entre otras, que el "régimen subsidiado de seguridad social en salud, entendido como un instrumento que promueve la igualdad de oportunidades para el acceso a prestaciones médico asistenciales a favor de aquellas personas que, en razón de sus condiciones de debilidad manifiesta, requieren de la especial protección del Estado, traducida en la ejecución de acciones afirmativas y tratamientos diferenciados positivos, como es el caso de los subsidios de salud...Para el caso específico de las demandantes, se advierte que a partir de sus condiciones socioeconómicas no es posible considerarlas como población objeto de la focalización del gasto público social, por lo que no serian, de manera general, beneficiarias del régimen subsidiado de seguridad social en salud..." NFT. 
absuelto por el representante de la autoridad eclesial, que

"la afiliación al régimen contributivo era una práctica usual dentro de las comunidades religiosas. Sin embargo, consideró pertinente aclarar que la legislación laboral vigente no definía $<$ la situación de los religiosos frente a su congregación en materia laboral>, salvo algunos pronunciamientos jurisprudenciales que niegan la existencia de una relación de trabajo entre la comunidad y los religiosos que la integran" 56 .

De igual forma, manifestó que cada una de las congregaciones, como la que presentó la acción de tutela, se regula por sus respectivas constituciones, equivalentes a los estatutos propios del sector privado ${ }^{57}$. Según lo indicado en el fallo y las consideraciones presentadas en el mismo, caben los siguientes interrogantes: ¿Cuántas personas vinculadas a entes jurídicos privados e inclusive públicos tales como asociaciones, fundaciones y corporaciones se encuentran amparadas bajo el régimen subsidiado de salud? ${ }^{58}$ ¿Qué tan efectivo es el SISBEN? ¿Qué cantidad de recursos estatales

\section{Ibíd.}

$57 \quad$ Ibíd. "Estos documentos prevén, a su vez, capitulos, definidos como la reunión de todos los miembros de la Congregación para la toma de decisión". Entre las resoluciones que adoptan los capitulos se encuentran las relacionadas con la seguridad social en salud de los miembros de la comunidad..las obligaciones consisten en la afiliación a seguridad social en salud y en la mayoría de los casos, afiliación a pensión, cuando la edad de la religiosa califica para tal requisito..." NFT. Cabe la pregunta: ¿y qué hacían entonces en el régimen subsidiado destinado para la población pobre y vulnerable?

58 No se puede olvidar que en muchas oportunidades los discursos políticos se centran el ofrecimiento de programas asistenciales para la población pobre y vulnerable, como si fueran los políticos los que "obsequian" dichos programas más no el Estado. De otra parte, hay que indicar que sólo hasta el 2005 se reguló la afiliación de las comunidades religiosas al SSSI, mediante el artículo 13 del Decreto $\mathrm{N}^{\circ} 3615$ del 10 de octubre de 2005 que indica que dichas comunidades se asimilarán a las asociaciones y tendrán el carácter de trabajadores independientes y no les será exigible la acreditación del número mínimo de afiliados. se pierden por el hecho de que personas que no deben estar vinculadas al Régimen Subsidiado de Salud lo están? La única respuesta cierta es que los perjudicados con esta serie de irregularidades, que afectan el SGSSS, son los miembros de la población pobre y vulnerable, por cuanto los recursos destinados de forma deficiente podrían ser reservados a aquellos.

\section{CONCLUSIONES}

De acuerdo a los últimos cambios al SGSSS en Colombia, cabe preguntar: ¿Se está privatizando la Seguridad Social en Salud en este país? El Artículo No. 14 de la Ley 1122 de 2007 (con evidentes influencias del Derecho Comercial), que regula todo lo relacionado con los actos ${ }^{59}$ y procedimientos médico-sanitarios, muestra claras señas de esa realidad. Más aún cuando la misma Corte Constitucional admite que "(...) al delegarse la prestación del servicio público de salud a una entidad particular, ésta ocupa el lugar del Estado para algo muy importante cual es la prestación de un servicio público; pero eso no excluye a que la entidad aspire a obtener una legitima ganancia (...)" 60 . E1 hecho de que las EPS privadas, como muestra, hayan aumentado su participación en el mercado con respecto al año 2004 al registrar una cobertura del $82.20 \%$ del total de los afiliados ${ }^{61}$, así lo indica. Con estas cifras, ¿Dónde está el estado de bienestar? E1 sistema de afiliación a la seguridad social en el Régimen Contributivo o Subsidiado ¿Se plasma mediante un simple contrato como el de arren-

59 MARTÍNEZ CALCERRADA, Luis. Derecho Médico, Tratado de Derecho Sanitario. Editorial Colex. Madrid. 1986. p. 69. Define lo atinente al Acto médico que se presenta como una "prestación o actividad del médico, que persigue, conforme a la técnica o arte correspondiente - la llamada lex artis ad hoc-, un efecto terapéutico o de curación de un enfermo o más genéricamente la promoción de la salud".

60 Corte Constitucional, sent. SU 480 del 25/11/97. M.P. Alejandro Martínez Caballero.

${ }^{61}$ Fuente: Asociación Colombiana de Medicina Integral - ACEMI - www.acemi.co u.v. 07/05/09 
damiento de un establecimiento de comercio, por ejemplo?

Es indiscutible, igualmente, que los procesos de información y clasificación de la población pobre y vulnerable en el país son improvisados. Su nivel de informalidad, la reglamentación no eficiente y el alto nivel de generalidad, traen como consecuencia que se encuentren amparados bajo el Régimen Subsidiado personas que no lo deberían estar, y lo que es más grave, que personas que requieren con urgencia pertenecer al dicho régimen no lo estén. Así las cosas, es relevante implementar los correctivos necesarios en el SISBEN, con el fin de focalizar de manera primigenia el gasto público social en el área de la salud, máxime cuando se administran, como en el país, recursos económicos bastante escasos. Para finalizar, es preciso que el Estado genere incentivos destinados a la población pobre y vulnerable, para que salgan de esta condición porque de lo contrario, se seguirá patrocinando tanto la mendicidad como la pobreza.

\section{BIBLIOGRAFÍA}

Asociación Colombiana de Medicina Integral - ACEMI www.acemi.org.co

CEPAL http://www.eclac.org/

Código de Comercio Colombiano.

Constitución Política de Colombia.

Corte Constitucional. Sentencia SU 480 del 25/11/97. M.P. Alejandro Martínez Caballero.

Corte Europea de Derechos Humanos http:// www.echr.coe.int/echr/Homepage

CORTÉS GONZÁLEZ, Juan Carlos. Reforma al sistema de salud. Legis. Bogotá. 2007.

CURREA, Lugo. La Salud como Derecho Humano. España. 2005.

Diario Oficial 46.506. Imprenta Nacional de Colombia.
E1 Tiempo. Artículo denominado: ¿Incoder Se Sumaría a la lista de las liquidadas? Sección Economía. Fecha de publicación: 21 de Abril de 2007 .

FRESNEDA, Oscar. Entrevista realizada al experto en Política Social en la revista Salud Colombia. Public Health Journal on the net. Edición 55, Febrero 2001. Disponible en: http://www. saludcolombia.com/actual/salud55/portad55. htm última visita: 26/04/09

GACETA del Congreso No. 596. 30 de noviembre de 2006.

GUILLEN, Raimon, et al. Diccionario Jurídico. Temis. Bogotá. 1990.

HERNÁNDEZ, Diego. La Universalidad comofundamento para el diseño dela política social. Disponible en: www.docentes.unal.edu.co/dfhernandezl/ docs/Universalism_SocialPolicy_DFHL.

http://sisconpes.dnp.gov.co/AYUDA/ CONSULTADEINFORMACI\%c3\%93N/ DocumentosConpesAprobados/tabid/131/ Default.aspx

Ley 100 de 1993.

Ley 319 de 1996.

Ley 715 de 2001.

Ley 1122 de 2007.

MARTINEZ CALCERRADA, Luis. Derecho Médico, Tratado de Derecho Sanitario. Editorial Colex. Madrid. 1986.

www.minproteccionsocial.gov.co

NÚÑEZ M, Jairo, et al. Evolución de las Politicas contra la Pobreza: De la previsión social a las transferencias condicionadas. Facultad Latinoamericana de Ciencias Sociales - FLACSO. Facultad de Economía. Universidad de los Andes. Bogotá. Documento CEDE 2006-31 issn 1657-7191 (edición electrónica) agosto de 2006.

OLANO GARCÍA, Hernán Alejandro. Constitución Política de Colombia. Ediciones Doctrina y Ley Ltda. Bogotá. 
Organización Mundial de la Salud www.oms. org

RODRÍGUEZ SALAZAR, Oscar, Crecimiento, equidad y ciudadania: Hacia un nuevo sistema de protección social. Universidad Nacional de Colombia, Facultad de Ciencias Económicas Centro de Investigaciones para del Desarrollo. Bogotá. 2006.

ROMERO, Coloma. La Medicina. España. 2002

Senado de la República de Colombia. Oficina de Información y Prensa. Comunicado 14 de noviembre de 2006.
Sentencia T-441/06 de 02/06/06. Expediente T- 1170394. Acción de tutela interpuesta por Ana Lucía Londoño Jaramillo y otras contra la Alcaldía Municipal de Manizales. M.P. Jaime Córdoba Triviño.

TAFUR, Luis Alberto. Controversia a la reforma de la Ley 100 de 1993, Ley 1122 de enero de 200. Corporación Editora Médica del Valle. Universidad del Valle. Cali. 2007.

Unidad Investigativa El Tiempo. Artículo "Tierra Movediza". Fecha de publicación: 11 de noviembre de 1997. 
